\title{
Regional Signals in Atmospheric and Oceanic Excitation of Polar Motion
}

\author{
Jolanta Nastula \\ Space Research Center of the PAS, Warsaw 00-716, Poland
}

Rui M. Ponte and David A. Salstein

Atmospheric and Environmental Research, Inc., Cambridge, MA 02139, USA

\begin{abstract}
Atmospheric and oceanic variability have been shown to play a role in the excitation of polar motion. Regional patterns of atmospheric and oceanic excitation are analysed and compared. The equatorial excitation functions, $\chi_{1}$ and $\chi_{2}$, for the ocean are computed using velocity and mass fields from a near-global ocean model, driven by observed surface winds stresses, surface heat and freshwater fluxes, for the period from January 1985 to June 1997. To understand the relative role of the ocean versus the atmosphere, we used atmospheric excitation functions computed from the National Centers for Environmental Prediction/National Center for Atmospheric Research reanalyses. We consider regional mass terms [bottom pressure and atmospheric surface pressure with the inverted barometer (IB) correction] and regional motion terms as well (currents and winds). Results here confirm recent findings that oceans supplement the atmosphere as an important source for polar motion excitation. Regional signals in the oceanic bottom pressure terms have comparable amplitudes to those in the atmospheric pressure-IB terms. The regional wind term amplitudes, however, are several times larger than the values for both regional oceanic currents term and atmospheric pressure-IB term. Power in regional oceanic excitation is distributed between seasonal and subseasonal timescales while in the case of atmospheric excitation it is concentrated rather at seasonal timescales.
\end{abstract}

\section{Introduction}

The links between atmospheric angular momentum (AAM) and polar motion have been studied in much detail in recent years because both observations of solid Earth fluctuations, taken by a number of space-based observing systems, and modern global atmospheric analyses available from the world's major weather centers are of sufficient accuracy for the purpose. The role of the atmosphere in driving variations in polar motion was demonstrated early on, but substantial discrepancies remained between atmospheric excitation and polar motion observations (see reviews by Eubanks 1993, Wilson 1995 and references therein). The importance of the oceans in explaining some of those discrepan- 
cies has been noted recently, after improvements in ocean modeling provided the capability to simulate the global ocean circulation. Recent findings show that oceanic angular momentum (OAM) signals are of sufficient magnitude to account for some of the existing equatorial angular momentum imbalances observed between Earth and atmosphere. For example, Ponte (1997) applied the formulation of Barnes et al. (1983) to velocity and mass fields from a numerical run of a constant-density, near-global model driven by twice-daily operational winds and atmospheric pressures to compute oceanic excitation of polar motion. This analysis was based on a relatively short time series (less than one year), but results showed that oceanic excitation levels were generally comparable to atmospheric excitation levels, at seasonal and shorter periods. The role of OAM in polar motion excitation was demonstrated by Ponte et al. (1998), with a OAM series covering more than 11 years (1985-1996). Using 5-day OAM calculation from a primitive equation model with full representation of thermodynamic processes, Ponte et al. (1998) showed that the addition of OAM to AAM values leads to significant improvements in the coherence between geophysical and observed polar motion, ranging from seasonal to 10 -day periods. Following the results of Ponte (1997) but using a longer 3-year series of the constant-density model Nastula \& Ponte (1999) confirmed the previous results at seasonal time-scales and extended the observable role of OAM signals in polar motion to periods as short as 5 days. A more recent analysis by Ponte \& Stammer (1999) has confirmed that the oceans can play a very important role in the Chandler, annual and semiannual wobble excitation, providing for much better amplitude and phase agreement with the observed excitation at these periods, in comparison with what is obtained when only the atmosphere is considered. Similar findings were reported by Celaya et al. (1999) based on a 50-year record from a climate model.

Although the important role of atmospheric and oceanic signals for polar motion excitation is documented, it is clear, however that even the combined atmospheric and oceanic data do not explain all of the observed polar motion signals. A comparison of the ratios between variances of the bandpass-filtered excitation functions in Table 1 shows that substantial parts of polar motion excitation still need to be explained, especially for periods shorter than 15 days.

It is illuminating to determine which regions in the ocean and atmosphere are acting as important sources of polar motion excitation, as a prelude to understanding the processes involved. This requires a decomposition of the oceanic and atmospheric excitation into their regional values. The regional variations of atmospheric excitation functions have already been computed in several papers. Salstein \& Rosen (1989) used a set of two-dimensional sectors to determine that subseasonal fluctuations of atmospheric mass in such areas as the North Atlantic, North Pacific, and the whole southern oceans strongly influence the global excitation functions for polar motion. Using an 8-year data set, Nastula (1997) partly confirmed that result. Some of the regions identified by Salstein $\&$ Rosen were found to be important, but interannual variations of polar motion were strongly coherent only with the pressure over midlatitude land areas (Nastula 1997, Nastula et al. 1997). When the inverted barometer (IB) relationship is included, spatial structure over the oceans is eliminated and temporal variability is much reduced. Thus, applying the IB correction to the pressure leads to the dominance of Eurasia and America instead, with nearly all South- 
Table 1. Ratio between variances of given pairs of $\chi_{1}$ and $\chi_{2}$ in three spectral ranges, for the period 1993-1995, according to the results from Table 2 by Nastula \& Ponte (1999).

\begin{tabular}{lccc}
\hline \hline & $2-15$ days & $15-150$ days & $>150$ days \\
\hline$\chi_{1}^{A+O} / \chi_{1}^{G}$ & $38 \%$ & $88 \%$ & $149 \%$ \\
$\chi_{2}^{A+O} / \chi_{2}^{G}$ & $42 \%$ & $49 \%$ & $61 \%$ \\
\hline
\end{tabular}

G: geodetic excitation function

$\mathrm{A}+\mathrm{O}$ : atmospheric + oceanic excitation functions

$\chi_{1}$ and $\chi_{2}$ describe the effective changes in the angular momentum components about two equatorial axes

ern Hemisphere contributions disappearing. Most recently Nastula \& Salstein (1999) using a much longer and more reliable data set expanded upon the earlier results and isolated most clearly the Eurasian, North American and other regions as important for exciting high-frequency polar motion with Eurasia especially prominent in this regard.

Although the analysis of regional characteristics of different parameters describing oceanic variability has a rich history, regional values of OAM have only recently been computed. Furuya \& Hamano (1998), based on Pacific Ocean analysis, found evidence for an important source of oceanic excitation in the midlatitude western North Pacific. Ponte \& Stammer (1999) using output from a near-global ocean model, isolated midlatitude regions $\left(30^{\circ}-70^{\circ}\right)$ as places of strong local oceanic excitation signals. The North Pacific basin was found to be generally more important for $\chi_{1}$ excitation while the South Pacific was important for both $\chi_{1}$ and $\chi_{2}$. The largest positive covariances of local with global signals occurred in the Kuroshio region near the western boundary of the North Pacific for $\chi_{1}$ and southwest of Australia for $\chi_{2}$.

With the knowledge of the important role of some atmospheric and oceanic region in driving polar motion, here we expand on Nastula \& Salstein (1999) and Ponte \& Stammer (1999) to compare regional patterns of atmospheric and oceanic excitation functions and to examine their relative amplitudes in different spectral bands.

\section{Data}

A commonly used technique for comparing the polar motion excitation with geophysical phenomena is through the determination of the so-called polar motion excitation functions, $\chi_{1}$ and $\chi_{2}$, describing the effective changes in the angular momentum components about two equatorial axes conventionally taken to point towards the Greenwich and $90^{\circ} \mathrm{E}$ meridians, respectively. These so-called geodetic excitation functions are compared to atmospheric and oceanic $\chi \mathrm{s}$ determined from appropriate geophysical observations and models. For this study 
we use two data sets of regional values of atmospheric and oceanic excitation functions of polar motion.

- Atmospheric excitation functions $\chi_{1}^{A}, \chi_{2}^{A}$ computed in 108 equal-area sectors by Nastula \& Salstein (1999) from four-times daily gridded data produced by the National Centers for Environmental Prediction/National Center for Atmospheric Research (NCEP/NCAR) reanalyses system (Salstein et al. 1993). The basic data include atmospheric surface pressure and the vertical distribution of the horizontal components of wind velocity on a $2.5^{\circ} \times 2.5^{\circ}$ latitude-longitude grid. To produce equal-area sectors we divide the globe, placing meridional boundaries every $30^{\circ}$ in longitude and zonal boundaries at $6.4,19.5,33.7,51.1$ and $90^{\circ}$ north and south (see Figure 1 in Nastula \& Salstein, 1999). Regional atmospheric functions were then averaged from four-times daily to a monthly frequency over the period from January 1985 to June 1997. As has been shown earlier, the atmospheric excitation functions, which are the sum of the pressure-IB and wind terms, are more correlated with the geodetic polar motion excitation function when the IB correction is included (see Eubanks 1993, Nastula 1995). Thus our atmospheric data contain pressure-IB and wind terms.

- Oceanic excitation functions $\chi_{1}^{O}, \chi_{2}^{O}$ computed by Ponte \& Stammer (1999) from the near-global ocean model, driven by observed surface winds stresses, surface heat and freshwater fluxes, for the period from January 1985 to June 1997. These regional OAM functions are based on oceanic bottom pressure and the horizontal component of oceanic currents determined on a $1^{\circ} \times 1^{\circ}$ latitude-longitude grid with monthly frequency. These oceanic data were averaged spatially into 108 sectors for consistency with the atmospheric data. The data are separated into bottom-pressure and currents terms.

\section{Results}

Perturbations of polar motion can be expressed by variations of the individual components or as the complex-valued $\chi=\chi_{1}+i \chi_{2}$. For estimation of $\chi^{A}=$ $\chi_{1}^{A}+i \chi_{2}^{A}$ and $\chi^{O}=\chi_{1}^{O}+i \chi_{2}^{O}$ variations we compute the standard deviation $(S D V)$ in every sector as

$$
S D V(\chi)=\sqrt{S D V\left(\chi_{1}\right)^{2}+S D V\left(\chi_{2}\right)^{2}} .
$$

We calculate latitude-longitude maps of the $S D V$ from two different time series that have been filtered using the Butterworth filter (Otnes \& Enochson, 1972) to include only 280-400 day periods (Figs. 1,2) and periods shorter than 150 days (Figs. 3,4 ). The spectral bands are defined as to give some insight into annual frequency (AF) and subseasonal frequency (SF) excitation, although the cut-off periods are arbitrary.

The spatial pattern of the $S D V$ of pressure-IB term is dominated by maxima over land areas (Figs. 1a, 3a), while for the bottom-pressure term the 

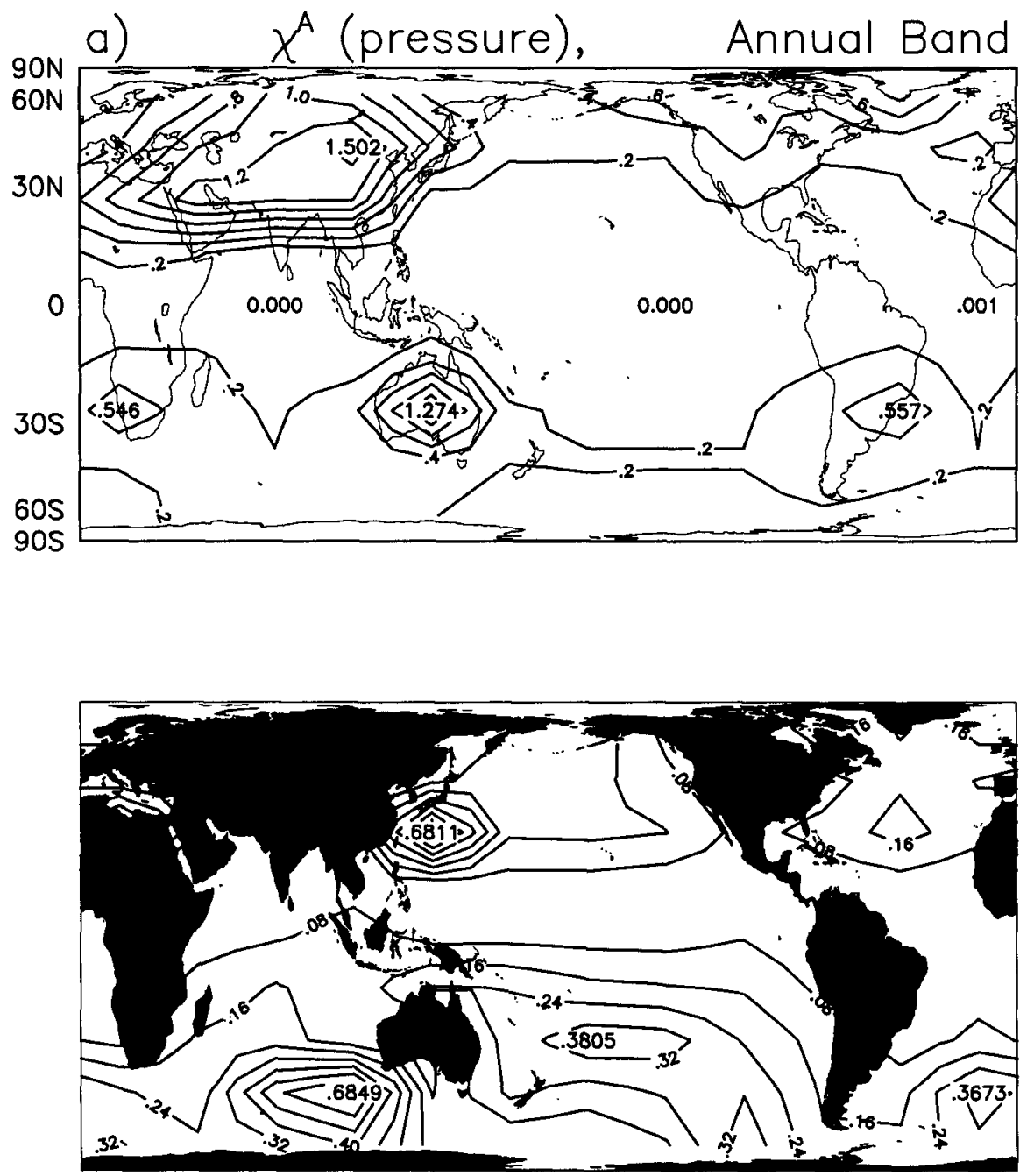

Figure 1. Maps of standard deviation $(S D V)$ of variations for (a) pressure-IB term of atmospheric excitation function $\chi^{A}$, (b) bottom pressure term of oceanic excitation function $\chi^{O}$, computed for annual frequency (AF) band. Variations with periods longer than 400 days and shorter then 280 days have been removed using a Butterworth band pass filter. Units are mas. 

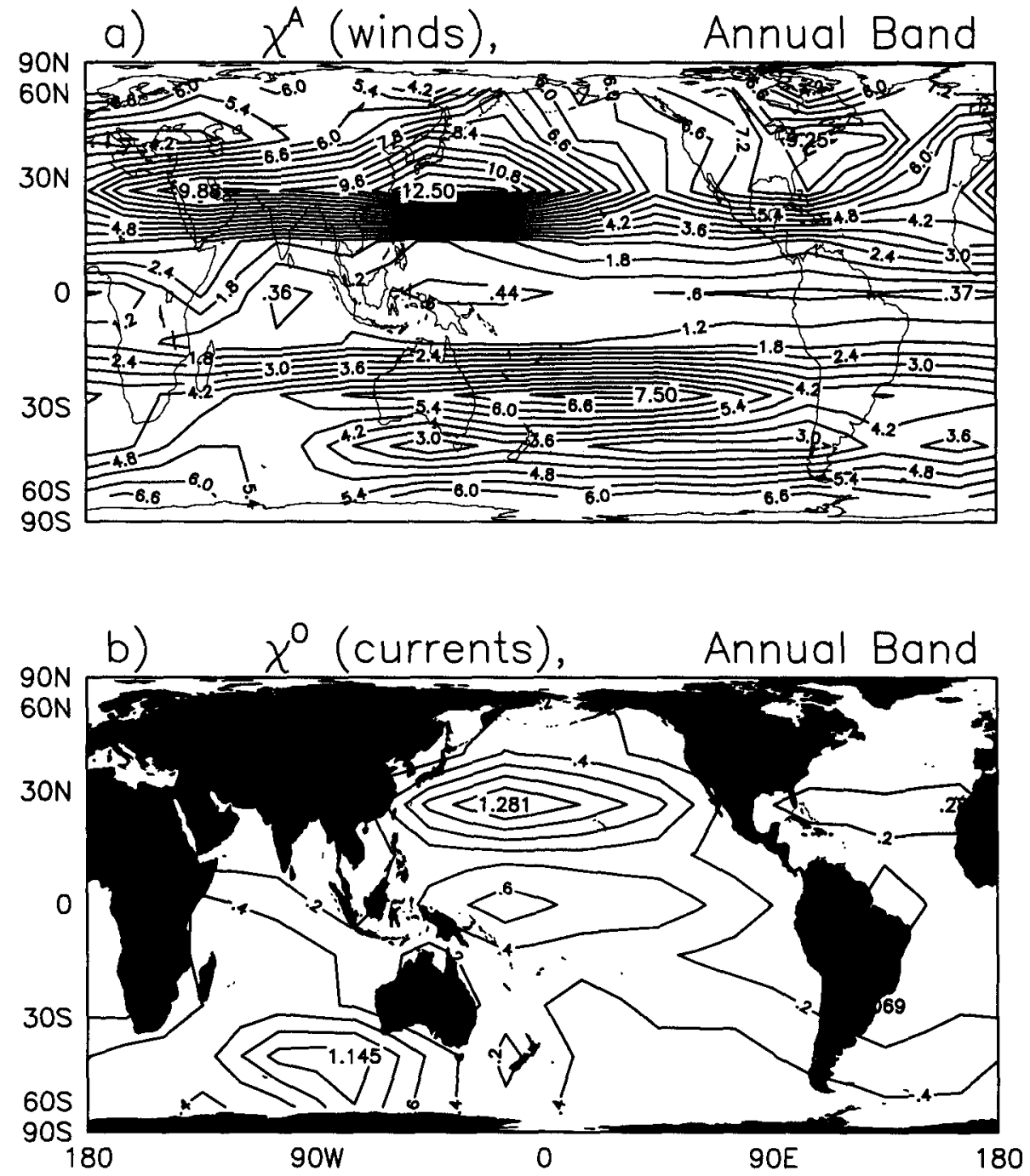

Figure 2. Maps of standard deviation $(S D V)$ of variations for (a) wind term of atmospheric excitation function $\chi^{A}$, (b) currents term of oceanic excitation function $\chi^{O}$, computed for annual frequency (AF) band. Variations with periods longer than 400 days and shorter then 280 days have been removed using a Butterworth band pass filter. Units are mas. 

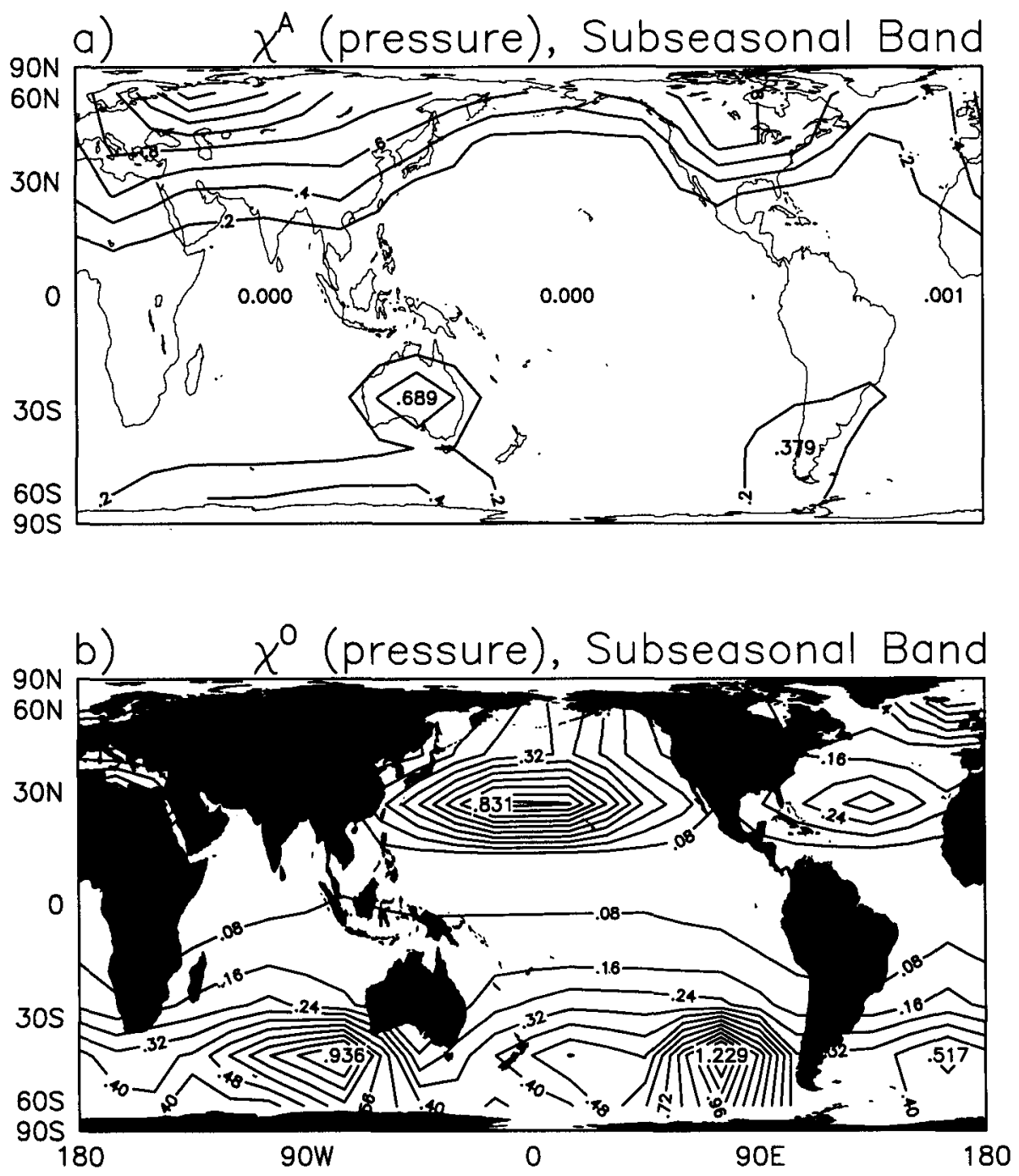

Figure 3. Maps of standard deviation ( $S D V$ ) of variations for (a) pressure-IB term of atmospheric excitation function $\chi^{A}$, (b) bottom pressure term of oceanic excitation function $\chi^{O}$, computed for subseasonal frequency (SF) band. Variations with periods longer than 150 days have been removed using a Butterworth high pass filter. Units are mas. 

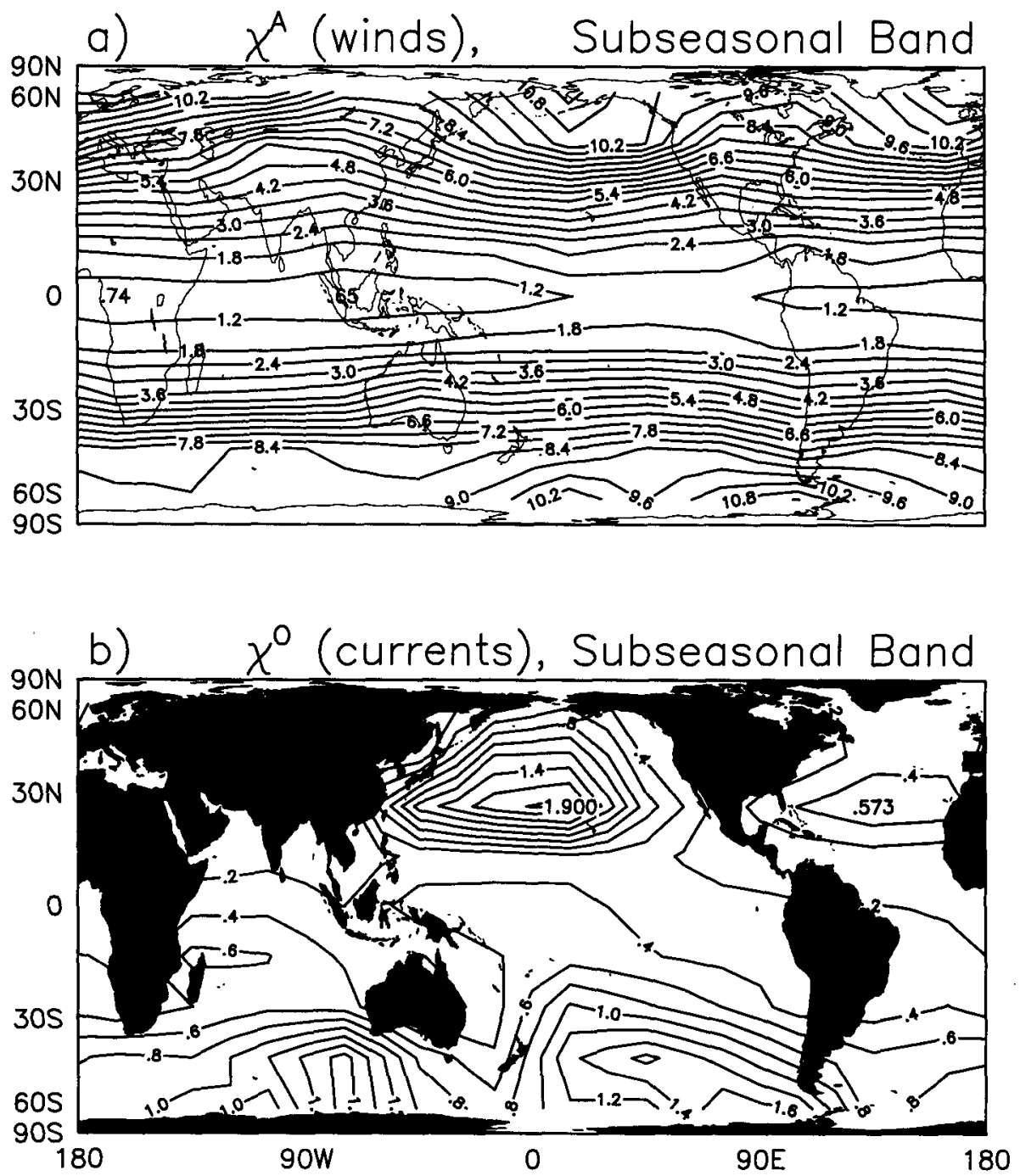

Figure 4. Maps of standard deviation ( $S D V$ ) of variations for (a) winds term of atmospheric excitation function $\chi^{A}$, (b) currents term of oceanic excitation function $\chi^{O}$, computed for subseasonal frequency (SF) band. Variations with periods longer than 150 days have been removed using a Butterworth high pass filter. Units are mas. 
strongest variability is centered over the midlatitude North Pacific and Southern Oceans (Figs. 1b, 3b). Maps of the $S D V$ of wind term have spatial patterns with maxima over the North Pacific and the North Atlantic and over the South Pacific associated with subtropical jet variability in both hemispheres (Figs. 2a, 4a). Oceanic excitation due to currents is strong in the North Pacific and the Southern Oceans (Figs. 2b, 4b).

The annual signals in the atmospheric pressure and oceanic bottom pressure terms, shown in Figure 1, are of the same order of magnitude but with $\chi^{A}$ being larger. The variations of those pressure terms in the subseasonal band, however, show different relations: $S D V$ s are still of the same order but with $\chi^{O}$ being larger. The regional $S D V$ s for the wind term are several times larger then the values for regional oceanic currents term in both spectral ranges (Figs. 2, 4). Comparing Figures $1 \mathrm{a}$ with $2 \mathrm{a}$ and $3 \mathrm{a}$ with $4 \mathrm{a}$, one can see that the wind term $S D V$ s are also generally larger than those for the atmospheric pressure term. It should be pointed out that when considering the global wind term its amplitudes are considerably smaller than those of the pressure term, even with the latter corrected for the IB (Nastula 1997, Nastula \& Salstein 1999). Thus we judge that the wind terms are rather noisy or out of phase regionally and analyses of the wind terms should be restricted to hemispheric or global terms.

Focusing on differences between the AF and the SF variability, the annual signal in atmospheric pressure term seems to be about ten times stronger than in the subseasonal band, while in the case of oceanic bottom pressure, signals in the annual and the subseasonal spectral bands have $S D V \mathrm{~s}$ of the same order with the subseasonal being larger. It is worth noting that the atmospheric winds and the oceanic currents appear to have a similar relation between $S D V$ s for the AF and the SF bands. The $S D V$ s for the wind term in the AF band are larger than those in the SF band while in the case of currents term the AF band signals have smaller $S D V$ s compared with those in the SF band. This implies that power in regional $\chi^{O}$ variability is distributed between seasonal and subseasonal bands while in the case of $\chi^{A}$ it is concentrated rather in the seasonal band.

\section{Closing remarks}

Comparison of regional distribution of AAM and OAM confirm that oceanic excitation is very important source of polar motion excitation. Bottom pressure variations in the ocean have comparable amplitudes to those of atmospheric pressure amplitudes (with IB) over land masses. Currents have comparably even larger amplitude than those of oceanic and atmospheric pressure regionally. Atmospheric wind excitation data, however, are quite variable regionally and require a more careful interpretation to be useful in studying polar motion variations in the various temporally filtered bands.

Atmospheric data are computed from observations processed through a data assimilation system while ocean data are related to models, driven only by boundary forcing. Thus the realism of present estimates of oceanic excitation of polar motion remains a concern. Determining which regions are the most important for excitation of polar motion may provide clues to the present accuracy of oceanic circulation models. 
Acknowledgments. The research reported here was supported by the Polish State Committee for Scientific Research through project 152/T12/97/13 and the U.S. National Science Foundation under project INT-9807014 (JN). The work of RP and DS was also sponsored by NASA's Earth Observing System Project under Grant NAG5-6309. Authors also thank Peter Nelson for technical assistance.

\section{References}

Barnes, R. T. H., Hide, R., White, A. A., \& Wilson. C. A. 1983, Proc. R. Soc. Lond., $A, \mathbf{3 8 7}, 31$.

Celaya, M. A., Wahr, J. M., \& Bryan, F. O. 1999, J. Geophys. Res., 104, 12813. Eubanks, T. M. 1993, in Contributions of Space Geodesy to Geodynamics: Earth Dynamics, Geodyn. Ser., 24, edited by D. Smith and D. Turcotte, AGU, Washington, D.C., 1.

Furuya, M. \& Hamano, Y. 1998, J. Geophys. Res., 103, 5493.

Nastula, J. 1995, Ann. Geophys., 13, 217.

Nastula, J. 1997, in Gravity, Geoid and Marine Geodesy, IAG Symp., 117, edited by J. Segawa et al., Springer-Verlag Berlin Heidelberg, 1997, 281.

Nastula, J., Kolaczek, B., \& Kosek, W. 1997, Ann. Geophys., 15, 1439.

Nastula, J. \& Ponte, R. M. 1999, Geophys. J. Int., 139, 123.

Nastula, J. \& Salstein, D. A. 1999, J. Geophys. Res., 104, 7347.

Otnes, R. K. \& Enochson, L. 1972, Digital time Series Analysis, John Wiley and Sons, New York.

Ponte, R. M. 1997, Geophys. J. Int., 130, 469.

Ponte, R.M., Stammer, D., \& Marshall, J. 1998, Nature, 391, 476.

Ponte, R. M. \& Stammer, D. 1999, J. Geophys. Res., 104, 22393.

Salstein, D.A. \& Rosen, R. D. 1989, J. Geophys. Res., 94, 9971.

Salstein, D.A., Kann, D. A., Miller, A.J., \& Rosen, R.D. 1993, Bull. Am. Meteor. Soc., 74, 67.

Wilson, C.R. 1995, Rev. Geophys., 33, suppl., 225. 